\title{
A Fast High-Order Solver for Problems of Scattering by Heterogeneous Bodies
}

\author{
Oscar P. Bruno and Alain Sei
}

\begin{abstract}
A new high-order integral algorithm for the solution of scattering problems by heterogeneous bodies is presented. Here, a scatterer is described by a (continuously or discontinuously) varying refractive index $n(x)$ within a two-dimensional (2-D) bounded region; solutions of the associated Helmholtz equation under given incident fields are then obtained by high-order inversion of the Lippmann-Schwinger integral equation. The algorithm runs in $\mathcal{O}(N \log (N))$ operations where $N$ is the number of discretization points. A wide variety of numerical examples provided include applications to highly singular geometries, high-contrast configurations, as well as acoustically/electrically large problems for which supercomputing resources have been used recently. Our method provides highly accurate solutions for such problems on small desktop computers in CPU times of the order of seconds.
\end{abstract}

Index Terms-Electromagnetic scattering, fast algorithms, highorder, inhomogeneous scatterer, penetrable scatterer.

\section{INTRODUCTION}

$\mathbf{T}$ HE calculation of electromagnetic scattering from electrically large two-dimensional (2-D) surfaces remains one of the most important and challenging problems in computational science. Roughly, these problems present difficulties as they require accurate descriptions and manipulation of highly oscillatory functions. Scattering problems involving 1-D integrals have been efficiently treated by means of high-order integrators (most notably the exponentially accurate trapezoidal-type rules [7], [9], [14], [16], [17]), which reduce dramatically the complexity necessary to meet a given accuracy requirement. In problems involving 2-D singular integrals, such as those considered here, the need for efficient methods is even more pressing; yet, few high-order algorithms have been proposed in this context, and limited success has thereby been achieved.

In this paper, we present a new high-order integral algorithm, see [19]. For simplicity, we do not consider here the 2-D integrals arising in scattering from 3-D objects. Instead, we restrict ourselves to the simplest electromagnetics problem in which 2-D singular integrals occur: scattering by a 2-D heterogeneous body under TE polarized radiation. A basic element in our method is the use of truncated Fourier expansions of the

\footnotetext{
Manuscript received May 25, 1999; revised November 25, 2002. This work was supported in part by the Air Force Office of Scientific Research, Air Force Materials Command, USAF, by Grants F49620-96-1-0008, F49620-98-1-0368, F49620-99-1-0010, and F49620-02-1-0049, and by the NSF under Contract numbers DMS-9596152, DMS-9523292, DMS-9816802, and DMS-0104531, and by the Powell Research Foundation.

O. P. Bruno is with the Department of Applied and Computational Mathematics, California Institute of Technology, Pasadena, CA 91125 USA.

A. Sei was with the Department of Applied and Computational Mathematics, California Institute of Technology, Pasadena, CA 91125 USA. He is now with the Northrop Grumman Space Technology, Redondo Beach, CA 90278 USA. Digital Object Identifier 10.1109/TAP.2003.818783
}

Green's function of the problem. The logarithmic point-singularities of the Green function manifest themselves, in the finite Fourier approximations, as singular arcs within the integration domain. As we shall show, such singularity distributions (together with appropriate smoothings, in case the refractive index exhibits discontinuities) allow for computation of the required 2-D integrals by means of simple 1-D high-order integrators. The algorithm is fast: it runs in $\mathcal{O}(N \log (N))$ operations, where $N$ is the number of discretization points. The sum of the Fourier expansions of the integrals together the GMRES matrix iterative solver, finally, deliver the desired high-order accuracy.

Although the Fourier series of the Green function converge slowly around the logarithmic singular points, use of truncated series as explained above does lead to accurate computations. Indeed, a Fourier expansion of the kernel of a given order gives rise to a Fourier expansion of the same order for the solution. Now, solutions are smoother than the Green's function: for a discontinuous scatterer, for example, the solution is continuous together with its first-order derivatives. It follows that the Fourier series for the solutions converge much faster than the corresponding ones for the scatterer, and thus low-order expansions of the Green function can provide very good approximations for solutions - in both the near and far fields. The order of the Green function expansion needs only to account for the oscillations in the incoming wave and those of the scatterer itself, as a rather interesting cancellation of errors produces good approximation for integrals from poor approximations for the kernel.

The concept of error cancellation mentioned above, which forms the basis of the algorithm presented in this paper, is illustrated in the Appendix by means of an elementary numerical integration example. In that example, an application of the trapezoidal rule to a truncated Fourier series of a discontinuous function results in numerical integrals with errors of the order of $h^{3}$. In fact, $\mathcal{O}\left(h^{3}\right)$ is precisely the order of accuracy our method in the most challenging case of discontinuous distributions of refractive-index; as shown in Section IV-D, for smoother scatterers much higher orders of convergence result. A full theoretical discussion of the convergence rates produced by our algorithm under various smoothness assumptions can be found in [4].

(A direct application of the methods presented in this paper to discontinuous scatterers for either TM or 3-D configurations, on the other hand, would yield the field exterior to the scatterer with an error of order $\mathcal{O}\left(h^{2}\right)$, since in such cases the normal derivatives of the solution are not continuous across surfaces of discontinuity of the refractive index. As shown in [5], however, the 
convergence rates of our method for all, TE, TM, and 3-D problems can be improved significantly by appropriate treatment of thin volumetric regions around surfaces where either discontinuities or reduced smoothness of the refractive index function occur.)

High-order methods have often been used in problems involving 1-D integrals [7], [14]; combinations of high-order 1-D integrators with fast summation techniques are given, for example, in [8], [17]. As for higher order methods considered earlier for problems related to 2-D singular integrals we mention [3], [10]. The approach in the first of these papers relies upon some form of refinement around the singular point. This technique uses a hierarchy of approximations of various orders to obtain, from a coarse grid calculation, results equivalent to those given by a low-order fine grid. This interesting approach exhibits excellent numerical properties for smooth kernels, and calculations with errors of order of $10^{(-9)}$ were reported in some cases. The accuracy of this algorithm is limited for singular integrals, however, as its discretizations do not resolve the kernel singularities to high order. As a result, applications of the method to singular problems exhibit the singularity enhancement associated with direct use of high-order approximation; for 2-D singular integrals errors of the order of $10^{(-4)}$ were reported.

In [10], on the other hand, the authors completely avoid explicit 2-D integrations. Instead, restricting themselves to calculation of solutions of Poisson's equation in a square (which can indeed be computed by means of a 2-D singular integral), they propose an algorithm which proceeds by finding solutions in small square elements as linear combinations of tensor products of Chebyshev polynomials. These basic elements are then pieced together to form a continuous solution on the square domain by means of fast multipole calculation of appropriate 1-D integrals. This method is extremely accurate, and it has produced full double precision solutions in fast desktop computations. The approach does rely heavily on the existence of a surface differential equation, which is generally not available, and it does not seem to extend easily either to integrations involving general singular kernels or to integration in general domains.

In sum, general high-order algorithms for problems associated with 2-D singular integrals have consistently found difficulties in the resolution of the high-order components of singularities and boundaries. Our approach addresses these difficulties through consideration of truncated Fourier expansions of the Green function of the problem. Such truncated expansions have the effect of redistributing the Green's function singularity into arcs inside the integration domain. The singularity in the 1-D integrals can then be resolved analytically, and it is, therefore, not necessary to refine the integrators near the singular points.

Our high-order algorithms can be applied to general configurations, including scatterers in which the refractive index varies discontinuously. Our approach is related to the well-known $k$-space method [2], which is based on representation of the the solution by its Fourier transform, in cartesian coordinates, in all of space. In this case, truncation of the Fourier transform at wavenumbers $K$ leads to errors of the order of $1 / K$, and thus, the error in the $k$-space method is of order one in the mesh-size (compare analysis and numerical results given in [18]). Our method takes advantage of the highly accurate approximations provided by Fourier series in the periodic case, and it thus achieves the claimed high-order accuracy.

In certain nontrivial cases the present algorithms have provided full double precision accuracies in 10-s desktop computations (on a 200-MHz SGI computer); for more challenging configurations, they yield engineering accuracies in short computing times as well as improved solutions with small additional efforts. An example is provided in Section IV-E of a highly singular scattering configuration containing cusps, for which solutions with errors of order $10^{(-5)}$ are obtained in a 13-s computation on the SGI computer aforementioned. A 2-s calculation on a 400-MHz PC, on the other hand, produces the solution for a scatterer nine-wavelengths in size with an error of $10^{(-3)}$. This problem has been treated recently [6], to the same order of accuracy, on a Cray C-90 computer. Thus, the performance of the present algorithm in general cases improves substantially over that exhibited by other methods available at present.

\section{PRELIMINARIES}

We consider a scattering configuration in which a bounded scatterer $\Omega$ is contained within a radius $R$ from the origin. The refractive index $n(x)$ varies arbitrarily in $\Omega$, and $n(x)=1$ outside the scatterer. Thus, setting $m(x)=1-n^{2}(x)$ we have

$$
\begin{aligned}
& n(x)=1 \text { and } m(x)=0 \text { for } x \text { outside } \Omega, \\
& \text { and, in particular, for }|x|>R .
\end{aligned}
$$

For a given incident field $u^{\text {inc }}$ with of wavelength $\lambda$ and wavenumber $\kappa=2 \pi / \lambda$, the total field $u$, equal to the sum of $u^{\text {inc }}$ and the scattered field $u^{s}$, is a solution of Helmholtz's equation

$$
\Delta u+\kappa^{2} n^{2}(x) u=0 .
$$

Together with conditions of radiation at infinity, this equation determines $u^{s}$ completely. Alternatively, $u$ can be obtained as the solution of the Lippmann-Schwinger integral equation

$$
u(x)=u^{\mathrm{inc}}(x)-\frac{i \kappa^{2}}{4} \int_{\Omega} H_{0}^{1}(\kappa|x-y|) m(y) u(y) d y
$$

where $H_{0}^{1}$ is the Hankel function of the first kind; see, e.g., [7]. We will solve this equation by an iterative technique which requires repeated evaluations of the right-hand side (RHS) of (3) for given inputs $u$. The accuracy of the calculation will be limited by that of the integral evaluations; thus, our focus on high-order quadrature rules for such singular integrals, see Section III.

We will be concerned, in particular, with calculation of farfield patterns. Denoting by $(a, \psi)$ the polar coordinates in the plane, these far fields are determined by the function $P$ in the expression

$$
u^{s}(a, \psi) \sim P(\psi) \sqrt{\frac{2}{\pi \kappa a}} e^{i\left(\kappa a-\frac{\pi}{4}\right)} \quad a \longrightarrow+\infty
$$


(cf. [1, p. 6]). We will compute the function $P$ from its Fourier and expansion

$$
P(\psi)=\sum_{\ell=-\infty}^{+\infty} \alpha_{\ell} e^{i \ell \psi}
$$

The coefficients $\alpha_{\ell}$ in this expansion can be obtained easily from the corresponding expansion for the scattered field

$$
u^{s}(a, \psi)=\sum_{\ell=-\infty}^{+\infty} B_{\ell}(a) e^{i \ell \psi}
$$

Indeed, by separation of variables we have $B_{\ell}(a)=c_{\ell} H_{\ell}^{1}(\kappa a)$ for some coefficients $c_{\ell}$. Consideration of the asymptotic form for the Hankel function readily gives $\alpha_{\ell}=(-i)^{\ell} c_{\ell}$, so that

$$
\alpha_{\ell}=(-i)^{\ell} \frac{B_{\ell}(a)}{H_{\ell}^{1}(\kappa a)} .
$$

\section{NUMERICAL METHOD}

Our algorithm is based on use of the addition theorem and high-order integration; its main lines are described in Section III-A. The high-order integrators require careful consideration of the integrand singularities. A discussion of such singularities and the overall integration strategies are presented in Sections III-B-E. Numerical tests and examples follow in Section IV.

\section{A. Addition Theorem}

As aforementioned, the addition theorem for the Hankel function (cf. [7])

$$
\begin{aligned}
H_{0}^{1}\left(\kappa\left|a e^{i \psi}-r e^{i \theta}\right|\right) & =\sum_{\ell=-\infty}^{+\infty} \mathcal{J}_{\ell}(a, r) e^{i \ell(\psi-\theta)} \\
\mathcal{J}_{\ell}(a, r) & =J_{\ell}(\kappa \min (a, r)) H_{\ell}^{1}(\kappa \max (a, r))
\end{aligned}
$$

provides a mean to deal with the singular behavior in the integrand of (3). To do this, we use polar coordinates $(a, \psi)$ and $(r, \theta)$

$$
x=a e^{i \psi} \quad y=r e^{i \theta}
$$

Then, in view of our convention (1), the integral on the RHS of (3) may be expressed in the form

$$
\begin{gathered}
K[u](a, \psi)=-\frac{i \kappa^{2}}{4} \int_{0}^{R} r d r \int_{0}^{2 \pi} H_{0}^{1}\left(\kappa\left|a e^{i \psi}-r e^{i \theta}\right|\right) \\
\times m(r, \theta) u(r, \theta) d \theta .
\end{gathered}
$$

Introducing the Fourier series expansions of $u^{\text {inc }}, u$, and $K[u]$

$$
\begin{aligned}
u^{\mathrm{inc}}(a, \psi) & =\sum_{\ell=-\infty}^{\infty} u_{\ell}^{\mathrm{inc}}(a) e^{i \ell \psi} \\
u(a, \psi) & =\sum_{\ell=-\infty}^{\infty} u_{\ell}(a) e^{i \ell \psi}
\end{aligned}
$$

$$
K[u](a, \psi)=\sum_{\ell=-\infty}^{\infty} K_{\ell}[u](a) e^{i \ell \psi}
$$

we then obtain

$$
K_{\ell}[u](a)=-\frac{i \kappa^{2}}{4} \int_{0}^{R} \mathcal{J}_{\ell}(a, r) r d r \int_{0}^{2 \pi} m(r, \theta) u(r, \theta) e^{-i \ell \theta} d \theta
$$

the integral equation is, thus, equivalent to the system of equations

$$
u_{\ell}(a)=u_{\ell}^{\mathrm{inc}}(a)+K_{\ell}[u](a)
$$

for the unknowns $u_{\ell}$.

Evaluation of quantities such as those on the RHS of (3) can be performed to high-order provided a high-order integrator is used for $K_{\ell}[u]$. (Indeed, since the Fourier series (8) for $K[u]$ converges fast, this quantity can be computed as the sum of a truncated expansion

$$
\sum_{l=-M}^{M} K_{\ell}[u](r) e^{i \ell \theta}
$$

for relatively small values of $M$.) As we will show, the singularities of the integrand of $K_{\ell}[u]$ do allow for high-order integration. Defining the linear map $A^{M}: v_{\ell} \longmapsto w_{l}$ given by

$$
v(r, \theta)=\sum_{\ell=-M}^{M} v_{\ell}(r) e^{i \ell \theta}
$$

and

$$
w_{\ell}=v_{\ell}-K_{\ell}[v], \quad l=-M \ldots M
$$

an application of the iterative solver GMRES to the linear problem $A^{M}[u]=u^{\text {inc }}$ will finally provide the desired high-order solution.

The case of discontinuous $m$ requires special consideration; the Fourier series of $m$

$$
m(a, \psi)=\sum_{\ell=-\infty}^{\infty} m_{\ell}(a) e^{i \ell \psi}
$$

will play a central role in this regard; see Section III-C.

In what follows, we will denote

$$
I_{\ell}(r)=\int_{0}^{2 \pi} m(r, \theta) u(r, \theta) e^{-i \ell \theta} d \theta
$$

so that

$$
K_{\ell}[u](a)=-\frac{i \kappa^{2}}{4} \int_{0}^{R} \mathcal{J}_{\ell}(a, r) I_{\ell}(r) r d r
$$




\section{B. Singularities}

An application of the addition theorem, as proposed in the previous section, leads to integration of functions whose singularities can be treated much more easily than those in (5).

The logarithmic singular point $x=(a, \psi)$ in (3) and (5) becomes a circular singular locus $r=a$ in each one of the integrands of (9) since the kernel $\mathcal{J}_{\ell}(a, r)$ have corner-type singularities (discontinuous derivatives) at $r=a$. An added near-singularity at $r=0$ occurs in (9), which consists of a logarithmic as well as polar $1 / r^{k}$ behavior $(k=-\ell,-\ell+2 \ldots)$. This is apparent from the representation (cf. [1, p. 51])

$$
H_{\ell}^{1}(z)=L(z) J_{\ell}(z)+D_{\ell}(z)
$$

for the Hankel function of order $\ell$, where, using

$$
\begin{aligned}
\psi(m)= & \sum_{s=1}^{m} \frac{1}{s}-\gamma \\
& \quad(\gamma=\text { Euler's Constant }=0.5772156649015329)
\end{aligned}
$$

we have denoted

$$
L(z)=\left(1+\frac{2 i}{\pi} \log \left(\frac{z}{2}\right)\right)
$$

and

$$
\begin{aligned}
D_{\ell}(z)= & -\frac{i}{\pi} \sum_{m=0}^{\ell-1} \frac{(\ell-m-1) !}{m !}\left(\frac{z}{2}\right)^{2 m-\ell} \\
& -\frac{i}{\pi} \sum_{m=0}^{\infty} \frac{\psi(m+\ell)+\psi(m)}{(-1)^{m} m !(\ell+m) !}\left(\frac{z}{2}\right)^{2 m+\ell} .
\end{aligned}
$$

While the point $r=0$ is not a true singularity of the kernel in (9), its near singular character described above needs nevertheless to be taken into account in the design of an integration rule, as it leads to large values for the derivatives of $\mathcal{J}_{\ell}(a, r)$ for values of $a$ and $r$ close to zero. Our specific strategy to treat this problem is explained toward the end of Section III-D.

\section{Angular Integration}

The fact that the function $m$ is discontinuous is the only obstacle to high-order integration in (13). To deal with such possible discontinuities we approximate $m$ by a truncated Fourier series

$$
m^{F}(a, \psi)=\sum_{\ell=-F}^{F} m_{\ell}(a) e^{i \ell \psi} .
$$

For finite $F, m^{F}$ provides a smooth approximation of $m$, and therefore, allows for high-order $\theta$-integration. Furthermore, as argued in the introduction and illustrated in Section IV, the solution associated with $m^{F}$ converges fast to the solution associated with $m$ as $F$ increases. As a result, high-order integration can be achieved even for discontinuous scatterers.

In accordance with the quantities $u, I_{\ell}$ and $K_{\ell}[u]$ of (7), (13), and (14), we define

$$
u^{M}(r)=\sum_{l=-M}^{M} u_{\ell}(r) e^{i \ell \theta}
$$

$$
I_{\ell}^{F, M}(r)=\int_{0}^{2 \pi} m^{F}(r, \theta) u^{M}(r, \theta) e^{-i \ell \theta} d \theta
$$

and

$$
K_{\ell}^{F, M}[u](a)=-\frac{i \kappa^{2}}{4} \int_{0}^{R} \mathcal{J}_{\ell}(a, r) I_{\ell}^{F, M}(r) r d r .
$$

The integrand defining $I_{\ell}^{F, M}(r)$ is a periodic regular function of $\theta$. Thus, the $\theta$ integration can be performed by means of the trapezoidal rule, which, in addition to being exponentially accurate, can be evaluated with a reduced operation count by means of the fast Fourier transform (FFT). Our integration problem now reduces to evaluation of radial integrals via high-order quadrature, cf. Section III-D.

It is important to note here that, unless the function $m$ is smooth within a circular domain, the function $m^{F}(r, \theta)$ is not a smooth function of $r$. It follows that $I_{\ell}^{F, M}(r)$ is also nonsmooth. For most discontinuous functions $m$ arising in practice, however, high-order integration of $I_{\ell}^{F, M}(r)$ in the radial variable is still possible. Indeed, assume the (possibly discontinuous) function $m$ is piecewise smooth, in the sense that $m$ is smooth in each one of a finite number of domains $\mathcal{D}_{1}, \ldots, \mathcal{D}_{s}$ with piecewise smooth boundary. Then, the singularities of $I_{\ell}^{F, M}(r)$ must occur either for values of $r$ for which the boundary of one of the domains $\mathcal{D}_{I}$ is nonsmooth or for values of $r$ for which the circle $C_{r}$ of radius $r$ is tangent to one of the domains $\mathcal{D}_{I}$. The type of the corresponding singularities of the function $I_{\ell}^{F, M}(r)$ can then be determined so as to allow for high-order integration through appropriate changes of variable.

For example, it can be seen that at a point $r_{0}$ for which $C_{r_{0}}$ is tangent to one of the domains $\mathcal{D}_{I}$, the singularity of the function $I_{\ell}^{F, M}(r)$ is of square-root type. More precisely, if $\mathcal{D}_{i}$ lies outside $C_{r_{0}}$, say, then this function is nonsmooth at $r=r_{0}$ from the right, but the new function $I_{\ell}^{F, M}\left(r_{0}+\left(\psi-r_{0}\right)^{2}\right)$ is smooth at $\psi=r_{0}$ from the right—as it follows easily from the assumption that the boundary of $\mathcal{D}_{i}$ is itself piecewise smooth. Thus, a quadratic change of variables can be used to remove the square-root behavior from the integrands. A change of variable that resolves the singular behavior in an interval $[a, b]$ containing one or possibly both endpoints as square root singularities can be constructed via manipulation of the function $r=r(\psi)$ given by

$$
\cos (\psi)=\left(\sqrt{\frac{\left(r^{2}-a^{2}\right)}{\left(b^{2}-a^{2}\right)}}\right)
$$

whose derivative vanishes at $\psi=0$ and $\psi=(\pi / 2)$. This transformation eliminates the singular behavior. The convergence rates resulting as the radial integrator is applied to the resulting functions is analogous to that resulting as the radial integrator is applied fully smooth scatterers.

(It is easy to convince oneself that, at tangency, the quantities $I_{\ell}^{F, M}(r)$ indeed have square-root type singularities. To see this, we first note that the Fourier coefficients $m_{\ell}(r)$ of $m^{F}$ (and $m$ ) are given by integrals on an integration domain which, for $r$ beyond tangency, excludes a certain interval around the tangency 
angle. Now, the equations of the endpoints of the excluded interval have square-root type singularities, so that the Fourier coefficients, and thus the function $m^{F}$ itself, inherit such singularities. In view of (20), finally, the same type of singularities are present in the function $I_{\ell}^{F, M}(r)$, as claimed.)

\section{High-Order Radial Integration}

To compute the integral in (21) to high order we first note that setting

$$
f(r)=I_{\ell}^{F, M}(r)
$$

and using (4) we have

$$
\begin{aligned}
K_{\ell}[u](a)= & H_{\ell}^{1}(\kappa a) \\
& \int_{0}^{\min (a, R)} J_{\ell}(\kappa r) f(r) d r \\
& +J_{\ell}(\kappa a) \int_{\min (a, R)}^{R} H_{\ell}^{1}(\kappa r) f(r) d r .
\end{aligned}
$$

In this section, we assume $f$ is differentiable to a sufficiently high order unless explicitly noted. As remarked at the end of the previous paragraph this is not a restrictive condition, since an appropriate change of variables can be used to resolve square-root type singularities that may occur in this function. We note that, under this assumption, the first integrand in (22) is smooth, while the second one contains logarithmic as well as polar singularities at the origin. (While the origin is not within the integration domain, these singularities do need to be resolved, as pointed out in Section III-B. This issue is addressed through appropriate moment computations, as indicated at the end of this section.)

Integration rules of order $p$ for integrals of the form

$$
\int_{r_{0}}^{r_{1}} J_{\ell}(\kappa r) f(r) d r
$$

to

$$
\int_{r_{0}}^{r_{1}} H_{\ell}^{1}(\kappa r) f(r) d r
$$

with smooth $f$ can be defined by sets of weights $v_{i}, w_{i}$ in such a way that, for example

$$
\int_{r_{0}}^{r_{1}} H_{\ell}^{1}(\kappa r) f(r) d r=\sum_{n} w_{n} f\left(r_{n}\right)+\mathcal{O}\left(h^{p}\right)
$$

with a similar formula for (23). Here, $h=\max \left\{\left|r_{i+1}-r_{i}\right|\right\}$, and the weights $v_{i}, w_{i}$ result from exact integration involving the Lagrange interpolating polynomials [11]. A high-order radial integrator for (22) can certainly be obtained, for any given value of $a$, as a combination of high-order integrators (25) in the regions $0 \leq r \leq a$ and $a \leq r \leq R$. A problem arises in using this approach in our context, however, as integrals of the type (22) must be computed for all values of $a$ in the radial integration mesh. Since for any given discretization few or no mesh points exist between the 0 and $a$ for small values of $a$ (or for integrals between $a$ and $R$, for $a$ close to $R$ ) it is not possible to use the available discretized data to integrate to high-order in such domains. Therefore, a different overall integration strategy is necessary in the present radial integration problem.

The integration algorithm we have devised for this problem is based on actual polynomial interpolations for the smooth functions associated with the integrands in (22). To evaluate $K_{\ell}[u]$ we divide the integration domain in a number $N_{i}$ of interpolation intervals $U_{1}, \ldots, U_{N i}$; the left and right endpoints of $U_{j}$ are denoted by $u_{j}^{0}$ and $u_{j}^{1}$. Each interval $U_{j}$, in turn, is provided with a grid of $N_{p}$ equi-spaced interpolation points $x_{j, k}, 1 \leq k \leq N_{p}$, with $x_{1, k}=u_{j}^{0}$ and $x_{N_{p}, k}=u_{j}^{1}$. Thus, the radial integral is discretized by an equispaced grid consisting of $N_{r}$ points, where

$$
N_{r}=N_{i}\left(N_{p}-1\right)+1 \text {. }
$$

To perform the integrations (23), (24), we define $P_{\ell}^{j}(r)$ and $Q_{\ell}^{j}(r)$ to be the polynomials of degree $N_{p}-1$ interpolating the smooth functions $f(r)$ and $J_{\ell}(\kappa r) f(r)$, respectively, at the points $x_{j, k}, 1 \leq k \leq N_{p}$. Then, denoting by $U_{j_{0}}$ the interpolation interval containing point $a$, the first integral in (22) is approximated by the sum of the integrals

$$
\sum_{j=1}^{j_{0}-1} \int_{u_{j}^{0}}^{u_{j}^{1}} Q_{\ell}^{j}(r) d r+\int_{u_{j_{0}}^{0}}^{a} Q_{\ell}^{j_{0}}(r) d r
$$

each one of which can be computed in closed form. (Care should be exercised when using such exact evaluations, however, as explained toward the end of this section.) Using (15), on the other hand, the second integral in (22) will be approximated by

$$
\begin{aligned}
& \int_{a}^{u_{j_{0}}^{1}} Q_{\ell}^{j_{0}}(r) L(r) d r+\sum_{j=1}^{j_{0}-1} \int_{u_{j}^{0}}^{u_{j}^{1}} Q_{\ell}^{j}(r) L(r) d r \\
& \quad+\int_{a}^{u_{j_{0}}^{1}} P_{\ell}^{j_{0}}(r) D_{\ell}(r) d r+\sum_{j=1}^{j_{0}-1} \int_{u_{j}^{0}}^{u_{j}^{1}} P_{\ell}^{j}(r) D_{\ell}(r) d r
\end{aligned}
$$

which can be computed in closed form as well. This provides a radial integration algorithm of order $\mathcal{O}\left(h^{p}\right)$, with $p=N_{p}$ if $N_{p}$ is even, and $p=N_{p}+1$ if $N_{p}$ is odd. An actual implementation of this approach requires interpolation and integration of polynomials, both of which give rise to numerical issues which require consideration, as described in what follows.

With regard to the interpolation problem, a number of possibilities are available; the algorithm we have chosen, routine polcof in [15], seems to be the one which suffers from the least amount of ill-conditioning. While it requires $N_{p}^{3}$ operations, a higher complexity than the $N_{p}^{2}$ operations required by other methods, its substantially better performance makes it preferable in our context. In any case, as shown in Section III-E, use of this method does not alter the order of magnitude of the overall complexity count of our algorithm. 
The integrations (27), (28) of polynomials multiplied by logarithms and powers also require some consideration. Indeed, the polynomials produced by the routine polcof mentioned above are functions of appropriately shifted variables. More precisely, this routine produces the interpolating polynomials $P$ and $Q$ in an interval $\left[r_{0}, r_{1}\right]$ as polynomials of the form $R=R(r-$ $t)$. Here, $t$ is a number that is not too far from the interval $\left[r_{0}, r_{1}\right]$-other choices lead to ill-conditioned numerics. Our usual choice is $t=r_{0}$.

Let us now consider the closed form integration of such shifted polynomials. In the case of the logarithmic integrands in (28), for example, the integrations reduce to evaluation of moments of the type

$$
\int_{r_{0}}^{r_{1}}(r-t)^{j} \log (r) d r .
$$

Expanding the power $(r-t)^{j}$, these moments could in turn be computed from the corresponding integrals of products $r^{k} \log (r)$, which can be obtained in closed form. This strategy leads to substantial cancellation errors, however, since the expansion of a binomial can produce a small number $(r-t)^{j}$ from subtractions involving large numbers arising from powers of $r$ and $t$. The alternative strategy we use is efficient, accurate and, in fact, quite simple: all the required moments are computed, at the beginning of each run, via Gaussian quadrature rules. This approach does not unduly increase the computational complexity as can be seen from the various numerical examples of Section IV (see, e.g., the normalized overhead NO in Table XII, which being essentially constant, shows that the overhead grows linearly with the size of the discretization). In addition, this method applies even for cases in which changes of variables are needed for smoothing, as explained in Section III-C.

\section{E. Complexity}

As before, we call $N_{\theta}$ and $N_{r}$ the number of points in the discretizations for the $\theta$ and $r$ variables, respectively; the total number of discretization points is, therefore, given by $N=$ $N_{r} N_{\theta}$. Computation of the quantity $I_{\ell}^{F, M}(r)$ of (20) for a given value of $r$ and for all required values of $\ell$ can be obtained with spectral accuracy in $N_{\theta} \log \left(N_{\theta}\right)$ operations. Indeed, this results from use of the trapezoidal rule, which yields such exponential convergence; the reduced operation count can be obtained, in turn, if the implementation takes advantage of the fact that the trapezoidal rule is algorithmically identical with certain FFTs. Thus, a total of $N_{r} N_{\theta} \log \left(N_{\theta}\right)$ operations produce all the necessary angular integrations. As for the radial integration, we note that each one of the necessary interpolations requires $N_{p}^{3}$ operations. Now, it is necessary to perform a number of the order of $N_{i}$ such interpolations for each one of the $M \leq N_{\theta}$ angular modes, so that the total interpolation cost can be estimated by $N_{i} N_{p}^{3} N_{\theta}=N N_{p}^{2}$. If we consider $N_{p}$ as fixed (this gives the order of the method), we see our algorithm requires a total operation count of the order of $N \log (N)$.

This fact is clearly illustrated in Table I, which shows computing times per GMRES iteration for the square scatterer of
TABLE I

COMPUTING TIMES FOR VARIOUS DISCRETIZATIONS AND CORRESPONDING

Time Ratios. As the Size of THE Discretization Is QuADRUPLED, THE TIME REQUiRED By ONE ITERATION IS MUlTIPLIED BY A FACTOR APPROXIMATELY EQUAL TO FOUR

\begin{tabular}{c|c|c|c|c}
\hline$M$ & $N_{\theta}$ & $N_{i}$ & Time/Iter (s) & Ratio \\
\hline 2 & 31 & 5 & 0.044 & \\
\hline 4 & 61 & 10 & 0.183 & 4.16 \\
\hline 8 & 121 & 20 & 0.608 & 3.32 \\
\hline 16 & 243 & 40 & 2.610 & 4.29 \\
\hline
\end{tabular}

TABLE II

COMPUTEd VALUES OF $\log _{2}\left(E\left(h / 2^{(\ell-1)}\right) / E\left(h / 2^{\ell}\right)\right)$ FOR COMPOSITE QuAdRATURE RULES USING INTERPOLANTS OF DEGREES $d=1$, $d=2$, AND $d=8$

\begin{tabular}{c|ccc}
\multicolumn{4}{c}{$\kappa R=1$} \\
\hline & $d=1$ & 2 & 8 \\
\hline$n=2$ & 2.01 & 4.05 & 9.65 \\
3 & 2.00 & 4.01 & \\
4 & 2.00 & 4.00 & \\
\hline
\end{tabular}

\begin{tabular}{c|ccc}
\multicolumn{4}{c}{$\kappa R=2$} \\
\hline & $d=1$ & 2 & 8 \\
\hline$n=2$ & 2.11 & 3.72 & 10.6 \\
3 & 2.02 & 3.98 & 9.89 \\
4 & 2.01 & 3.99 & \\
\hline
\end{tabular}

\begin{tabular}{c|ccc}
\multicolumn{4}{c}{$\kappa R=4$} \\
\hline & $d=1$ & 2 & 8 \\
\hline$n=2$ & 2.60 & 5.11 & 11.6 \\
3 & 1.98 & 4.01 & 9.76 \\
4 & 1.99 & 4.00 & 8.16 \\
\hline
\end{tabular}

\begin{tabular}{c|ccc}
\multicolumn{4}{|c}{$\kappa R=8$} \\
\hline & $d=1$ & 2 & 8 \\
\hline$n=2$ & & 4.77 & 9.11 \\
3 & & 5.51 & 10.98 \\
4 & 2.05 & 4.08 & 9.72 \\
\hline
\end{tabular}

Fig. 4. According to Table I, an increase by a factor of four in the total number $N=N_{r} N_{\theta}=N_{i} N_{p} N_{\theta}$ of discretization points leads to an increase by a factor approximately equal to 4 in the time per iteration - in agreement with our operation count. Note the accompanying increases in the truncation parameter $M$ of (19) which are necessary to obtain improved accuracies from the finer discretizations.

\section{NumERICAL RESUlTS}

In this section, we present a variety of convergence tests and demonstrations of the capabilities of our algorithm. Thus, in Sections IV-A and IV-B, we focus on the convergence properties of the radial and angular integrators. In Section IV-C, in turn, we demonstrate the performance of our solver on nonsmooth scatterers. Some of these examples involve scatterers containing constant refractive indexes. Naturally, the present solver is not intended for treatment of homogeneous bodies, which are often handled more efficiently by boundary integral methods. The homogeneous examples are only used as a check of correctness and accuracy; a number of other configurations, containing smooth as well as nonsmooth heterogeneous scatterers, are also considered below. In Sections IV-D and IV-E, finally, we present computations involving a large inhomogeneous scatterer and a complex body containing cusps. The computations reported in Sections IV-A, C and E were performed on a 200-MHz Silicon Graphics R-10 000 desktop computer; the ones in Sections IV-B and D resulted from computations performed on a 400-MHz PC, except for Table VIII, which was performed on a 700-MHz PC. In all the tables in this section, the quantity "far field error" was computed as the maximum absolute value of the difference between the computed and "exact" far field. As detailed in the text, the "exact" values of the far 

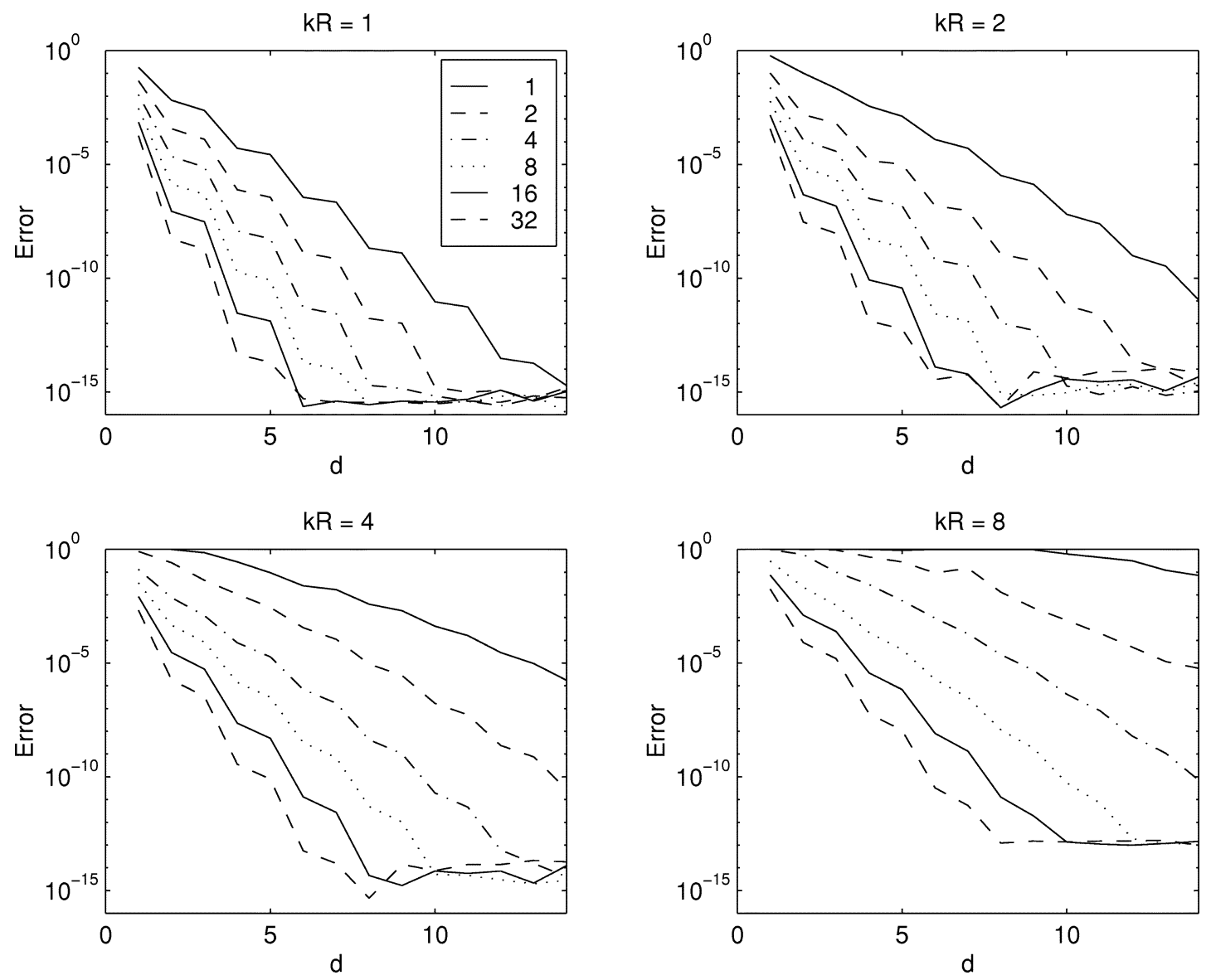

Fig. 1. Convergence of composite quadrature rules for various values of $\kappa R$. Figures show the error in the far fields for radial approximations with polynomials of degree $d=N_{p}-1$, and with $N_{i}=1,2, \ldots, 32$.

field were computed from an exact solution in some cases, and from an application of our own numerical method with a very fine discretization, in other cases. The quantity "ratio," which is displayed in several of the tables in this section, equals the quotient of errors displayed in two consecutive rows.

\section{A. Convergence Tests: Radial Integration}

Our first test concerns convergence rates for the radial integrator, for which error bounds follow from the theory of polynomial approximation. In what follows, we call $E_{d}(h)$ the error of the radial algorithm of Section III-D using polynomials of degree $d$ on a radial grid of spacing $h$. Based on approximation theory one can establish the asymptotic identities

$$
\log _{2}\left(\frac{E_{d}\left(\frac{h}{2^{\ell(\ell-1)}}\right)}{E_{d}\left(\frac{h}{2^{\ell}}\right)}\right) \approx \begin{cases}d+2, & \text { if } d \text { even } \\ d+1, & \text { if } d \text { odd }\end{cases}
$$

which display the order of convergence of the integrator as equal to $\mathcal{O}\left(h^{d+2}\right)$ or $\mathcal{O}\left(h^{d+1}\right)$, depending on the parity of $d$. Such error estimates manifests themselves clearly in the corresponding accuracies for the computed solutions-as we show in what follows. For our tests, we used a homogeneous circular cylinder of radius 1 with constant index of refraction $n=\sqrt{2}$ under plane wave incidence along the $x$ axis. The corresponding solutions and far-field patterns are given by well known expressions [12, p. 301] which we use in the following error evaluations. Since we only wish to test the radial integrator at this stage, for the examples in this section we used a fixed and sufficiently fine angular mesh so as to ensure double precision accuracy in all the relevant angular integrals.

Table II shows the calculated values of the quantity on the left-hand side of (29) for various values of $\kappa R$ and various values of $d$. The approximate agreement of these calculated values with the right hand side of (29) shows that the theoretical order of convergence of the radial algorithm is in fact achieved. The empty entries correspond to cases in which either the asymptotic regime has not been reached or the machine precision has been exceeded. In Fig. 1, on the other hand, we plot the maximum absolute values of the difference of exact and computed far-field patterns for the circular cylinder as a function of $d$. These graphs show that, as expected, high-order integration can lead to very accurate numerics with rather coarse discretizations. The staircase nature of these curves is explained by the parity dependence (29) of the error.

\section{B. Convergence Tests: Angular Integration}

As we have claimed, our Fourier-smoothing approach gives rise to high-order convergence even for discontinuous refractive index distributions, and to even higher order convergence for smoother scatterers. In order to demonstrate the convergence of the method as the angular mesh and the Fourier approximations are enlarged, we use a fixed and very fine radial discretization. 
TABLE III

SCATTERING BY A SOUARE; $\kappa R=1$

\begin{tabular}{c|c|c|c|c}
\hline$M$ & $F$ & $N_{\theta}$ & Far Field Error & Ratio \\
\hline 4 & 4 & 8 & $3.7 \cdot 10^{(-4)}$ & \\
\hline 8 & 8 & 16 & $3.0 \cdot 10^{(-5)}$ & 12.3 \\
\hline 16 & 16 & 32 & $3.3 \cdot 10^{(-6)}$ & 9.1 \\
\hline 32 & 32 & 64 & $3.8 \cdot 10^{(-7)}$ & 8.7 \\
\hline
\end{tabular}

TABLE IV

SCATTERING BY A SQUARE; $\kappa R=5$

\begin{tabular}{c|c|c|c|c}
\hline$M$ & $F$ & $N_{\theta}$ & Far Field Error & Ratio \\
\hline 16 & 16 & 32 & $2.4 \cdot 10^{(-3)}$ & \\
\hline 32 & 32 & 64 & $3.2 \cdot 10^{(-4)}$ & 7.5 \\
\hline 64 & 64 & 128 & $4.1 \cdot 10^{(-5)}$ & 7.8 \\
\hline 128 & 128 & 256 & $5.1 \cdot 10^{(-6)}$ & 8.0 \\
\hline 256 & 256 & 512 & $5.7 \cdot 10^{(-7)}$ & 8.9 \\
\hline
\end{tabular}

TABLE V

SCATTERING BY THE Two-LAYER CiRCULAR CYLINDER DEFINED IN (30); $\kappa=1$. ERRORS COMPUTED BY COMPARISON WITH THE EXACT SOLUTION

\begin{tabular}{c|c|c|c|c}
\hline$M$ & $F$ & $N_{\theta}$ & Far Field Error & Ratio \\
\hline 2 & 0 & 4 & $5.3 \cdot 10^{(-3)}$ & \\
\hline 4 & 0 & 8 & $3.2 \cdot 10^{(-7)}$ & $1.6 \cdot 10^{4}$ \\
\hline 8 & 0 & 16 & $1.5 \cdot 10^{(-13)}$ & $2.1 \cdot 10^{7}$ \\
\hline
\end{tabular}

Our first example in this section concerns scattering by a square (see Fig. 4). Tables III and IV show the convergence of the solution as the number $F$ of Fourier modes used for Fourier smoothing of this discontinuous scatterer is increased. The errors in Tables III and IV were computed by comparison with the numerical solution for a much finer mesh. We see that, consistent with cubic convergence, the error ratios resulting from doubling of the angular mesh and the Fourier parameter $F$ are approximately equal to eight. An example in which errors for a discontinuous scatterer are computed by comparison with an exact solution is presented in the following section.

We next include a classical test case for inhomogeneous-scatterer solvers: the two-layer circular cylinder. Here we consider a cylinder of radius one, with index of refraction defined by

$$
n^{2}(x, y)=\left\{\begin{array}{ll}
4 & 0 \leq x^{2}+y^{2}<\frac{1}{2} \\
16 & \frac{1}{2} \leq x^{2}+y^{2} \leq 1
\end{array} .\right.
$$

Numerical results are presented in Tables V-VII; here errors were computed by comparison with the exact solution. Fig. 2, in turn, contains a graphical comparison with the exact solution. Although the function $n^{2}(x, y)$ is discontinuous, the convergence of our algorithm is much faster than cubic in this case. This can be understood easily: the discontinuities in this distribution of refractive index do not give rise to discontinuities in the $\theta$ variable for any value of $r$. If follows that all the integrations performed involve only smooth functions, and, thus, very high-order convergence results.

A point of general relevance with regard to the angular integration is that its influence on the overall complexity of the method is mostly determined by the number $M$ of modes used to truncate the solution, see (11), rather than the truncation pa-
TABLE VI

SCATtering By the Two-Layer CirCUlar CyLinder DeFined IN (30); $\kappa=2$. ERRORS COMPUTED BY COMPARISON WITH THE EXACT SOLUTION

\begin{tabular}{c|c|c|c|c}
\hline$M$ & $F$ & $N_{\theta}$ & Far Field Error & Ratio \\
\hline 4 & 0 & 8 & $4.3 \cdot 10^{(-3)}$ & \\
\hline 8 & 0 & 16 & $9.1 \cdot 10^{(-11)}$ & $4.7 \cdot 10^{7}$ \\
\hline 16 & 0 & 32 & $1.0 \cdot 10^{(-13)}$ & $9.1 \cdot 10^{2}$ \\
\hline
\end{tabular}

TABLE VII

SCATtering By the Two-Layer CirCUlar Cylinder DeFined IN (30); $\kappa=4$. ERRORS COMPUTED BY COMPARISON WITH THE EXACT SOLUTION

\begin{tabular}{c|c|c|c|c}
\hline$M$ & $F$ & $N_{\theta}$ & Far Field Error & Ratio \\
\hline 4 & 0 & 8 & $\left.0.9 \cdot 10^{(-0}\right)$ & \\
\hline 8 & 0 & 16 & $\left.1.5 \cdot 10^{(-3}\right)$ & $6.0 \cdot 10^{2}$ \\
\hline 16 & 0 & 32 & $8.0 \cdot 10^{(-13)}$ & $1.9 \cdot 10^{9}$ \\
\hline
\end{tabular}

TABLE VIII

DEPENDENCE OF COMPUTING TIMES ON $F$ AND $N_{\theta}$. SCATTERING BY A SQUARE; $\kappa R=5$. RUNS PERFORMED ON A 700-MHz PC

\begin{tabular}{c|c|c|c|c}
\hline$M$ & $F$ & $N_{\theta}$ & Far Field Error & Tot. Time(s) \\
\hline 64 & 64 & 128 & $4.1 \cdot 10^{(-5)}$ & 91 \\
\hline 64 & 64 & 256 & $4.4 \cdot 10^{(-5)}$ & 93 \\
\hline 64 & 128 & 256 & $4.1 \cdot 10^{(-5)}$ & 93 \\
\hline 64 & 256 & 512 & $4.1 \cdot 10^{(-5)}$ & 96 \\
\hline 64 & 512 & 1024 & $4.1 \cdot 10^{(-5)}$ & 101 \\
\hline
\end{tabular}

rameter $F$ or the number $N_{\theta}$ of angular discretization points used. This fact is demonstrated in Table VIII; we see that an increase by a factor of two or even four in $N_{\theta}$ does neither give rise to significant increases in computing time nor to changes in accuracy, so that, indeed, the most important complexity-controlling parameters are $M$ and $N_{r}$. (We note that increases in $F$ beyond those required by a certain accuracy must be accompanied by corresponding increases in $N_{\theta}$ if the given error level is to be maintained.) Taking $N_{\theta}$ to equal the minimum value for which a prescribed accuracy is attained, then we find that $M$ is proportional to $N_{\theta}$; see Tables III, through VII above. In what follows we, therefore, make reference to the parameter $M$ rather than $N_{\theta}$. As illustrated in Table XII, the overall complexity of the method is linear in the product $M N_{r}$.

\section{Nonsmooth Objects: Comparison With an Exact Solution}

Any scatterer which is not described by a smooth variation of the refractive index within a circle centered at the origin must be considered as a nonsmooth scatterer within our framework. Fortunately, the circular geometry itself provides excellent means to assess the performance of our methods in such cases. To do this, it suffices to consider a circular scatterer centered at a point other than the origin. For our example, we thus consider the circle of radius 1 centered at the point $(2,0)$ on the real axis, as shown in Fig. 3. With reference to Section III-A and Section III-C, we see that the function $m$ characterizing this scatterer is discontinuous in the integration domain $\{r \leq R=3\}$. Note that, for any given $F$, the approximating function $m^{F}$ takes nonzero values almost everywhere in a corona of width 2 .

In Table IX, we present the errors in our calculations of both the near and far fields for this geometry. Here, as well as in 


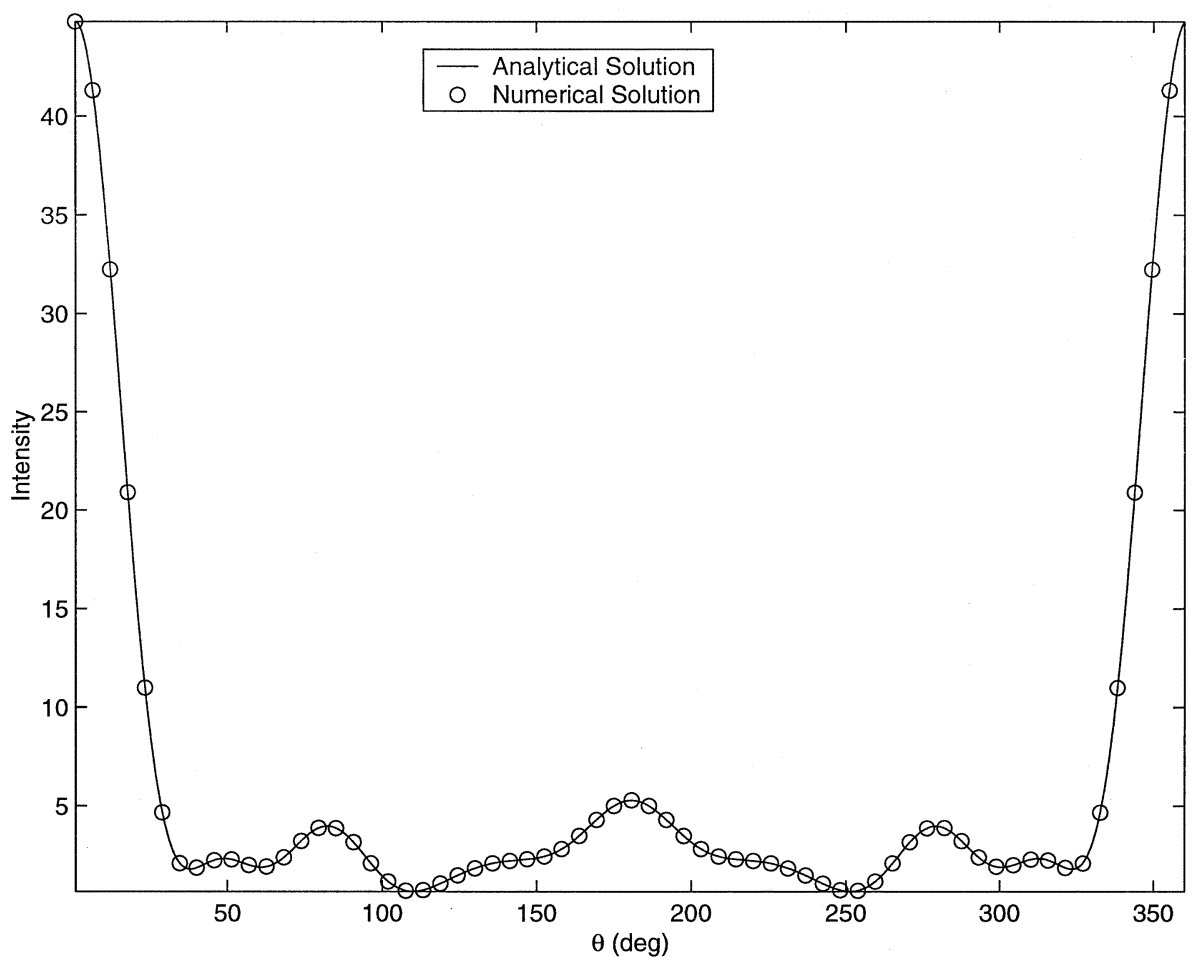

Fig. 2. Far-field (intensity) scattered by the two-layer circular cylinder defined in (30); $\kappa=4$.

TABLE IX

TimingS AND ERRors For the CONFIGURATION OF FIG. $3 ; N_{p}=3$

$$
\kappa R=3
$$

\begin{tabular}{c|c|c|c|c|c|c}
\hline$M$ & $N_{r}$ & \# Iter & Time/Iter (s) & Total Time (s) & $\begin{array}{c}\text { Error } \\
\text { (Interior) }\end{array}$ & $\begin{array}{c}\text { Error } \\
(\text { Far Field) }\end{array}$ \\
\hline 10 & 13 & 2 & 1.07 & 2.86 & $4 \cdot 10^{(-3)}$ & $2 \cdot 10^{(-4)}$ \\
\hline 20 & 21 & 2 & 2.03 & 5.40 & $6 \cdot 10^{(-4)}$ & $3 \cdot 10^{(-5)}$ \\
\hline 40 & 43 & 2 & 6.07 & 16.0 & $4 \cdot 10^{(-5}$ ) & $4 \cdot 10^{(-6)}$ \\
\hline 80 & 83 & 2 & 20.8 & 54.46 & $1 \cdot 10^{(-5)}$ & $7 \cdot 10^{(-7)}$ \\
\hline
\end{tabular}

$\kappa R=6$

\begin{tabular}{c|c|c|c|c|c|c}
\hline$M$ & $N_{r}$ & \# Iter & Time/Iter (s) & Total Time (s) & $\begin{array}{c}\text { Error } \\
\text { (Interior) }\end{array}$ & $\begin{array}{c}\text { Error } \\
\text { (Far Field) }\end{array}$ \\
\hline 10 & 17 & 4 & 1.33 & 6.20 & $3 \cdot 10^{(-2)}$ & $1 \cdot 10^{(-2)}$ \\
\hline 20 & 25 & 6 & 2.45 & 16.30 & $3 \cdot 10^{(-3)}$ & $7 \cdot 10^{(-4)}$ \\
\hline 40 & 41 & 8 & 5.52 & 47.87 & $3 \cdot 10^{(-4)}$ & $8 \cdot 10^{(-5)}$ \\
\hline 80 & 83 & 10 & 22.22 & 235.46 & $3 \cdot 10^{(-5)}$ & $6 \cdot 10^{(-6)}$ \\
\hline
\end{tabular}

Section IV-E we used $d=2\left(N_{p}=3\right)$, since higher order methods did not lead to an improved performance in these nonsmooth cases. (In detail, note that, for a discontinuous scatterer, the angular integration rule we use is accurate to order 3 , and thus it is not necessary to use radial integration of order higher than three to preserve an overall third-order convergence. Use of even higher order radial integration rules do give rise to correspondingly higher order convergence for the radial integrator (as shown in Fig. 1), and for very high accuracies they would eventually be preferable to the third-order radial interpolations used in the text. However, as is known, the integration errors do not depend on the high-order rule only - they also depend on the particular function being integrated. It so happens that, for tolerances of single precision or lower, the $N_{p}=3$ radial integration rule we use produces the results within the prescribed

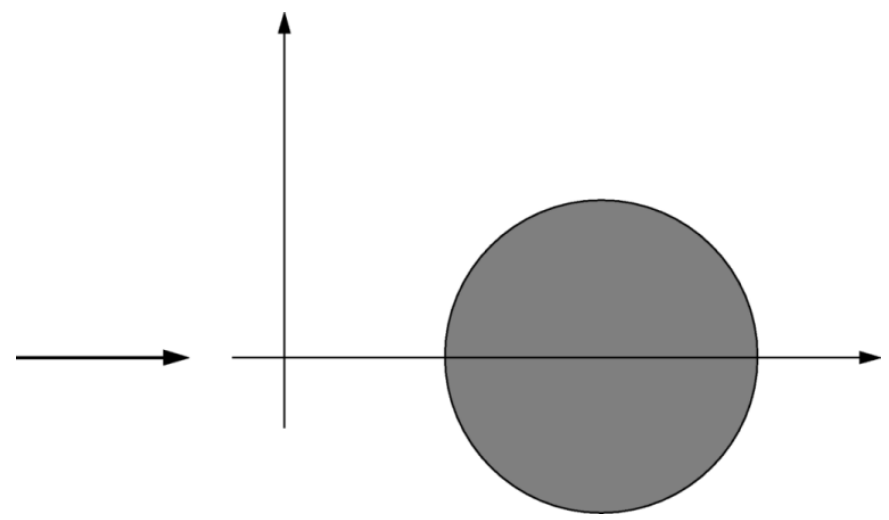

Fig. 3. A test: Exact geometry treated as a nonsmooth object.

tolerances in the least time, and thus our our choice of such radial integrator.) Note that, in accordance with (29), the choice $d=2$ produces a method of order 4 . The error in the interior of the circle was calculated as the maximum difference, in absolute value, between the calculated values and the values given by the exact solution [12]. The far field error, on the other hand, is the maximum absolute value of the difference between the calculated and exact far-field functions $P$ of Section II. This example thus demonstrates the applicability of our algorithm through comparison with an exact solution, both in the near and far fields, for cases in which the refractive index (and, therefore, the functions $m$ ) is discontinuous within the integration domain. Additional applications to nonsmooth scatterers are given in the following sections; in those cases errors were computed by comparison with a highly resolved calculation; a performance similar to the one shown in Table IX was obtained in those cases as well. 


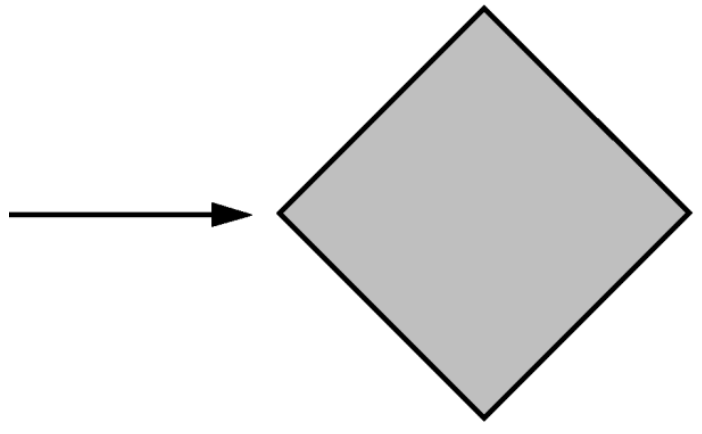

(a)

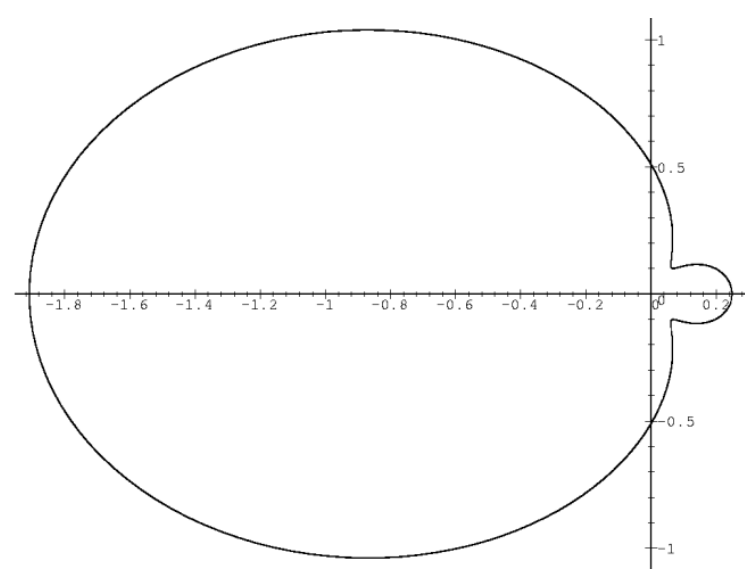

(b)

Fig. 4. Left: A scattering configuration containing corners; Square of side $=\sqrt{2}$ with refractive index $n=\sqrt{2}$. Right: associated far-field pattern $\kappa R=2$.

TABLE $\mathrm{X}$

ERRORS FOR THE SQUARE SCATTERER OF FIG. 4. $\kappa R=2 ; N_{p}=3$

\begin{tabular}{c|c|c|c}
\hline$M$ & $N_{p}$ & $N_{i}$ & Far Field Error \\
\hline 2 & 3 & 5 & $1.1 \cdot 10^{(-2)}$ \\
\hline 4 & 3 & 11 & $2.4 \cdot 10^{(-3)}$ \\
\hline 8 & 3 & 18 & $1.9 \cdot 10^{(-4)}$ \\
\hline 16 & 3 & 25 & $1.8 \cdot 10^{(-5)}$ \\
\hline 32 & 3 & 33 & $1.1 \cdot 10^{(-6)}$ \\
\hline
\end{tabular}

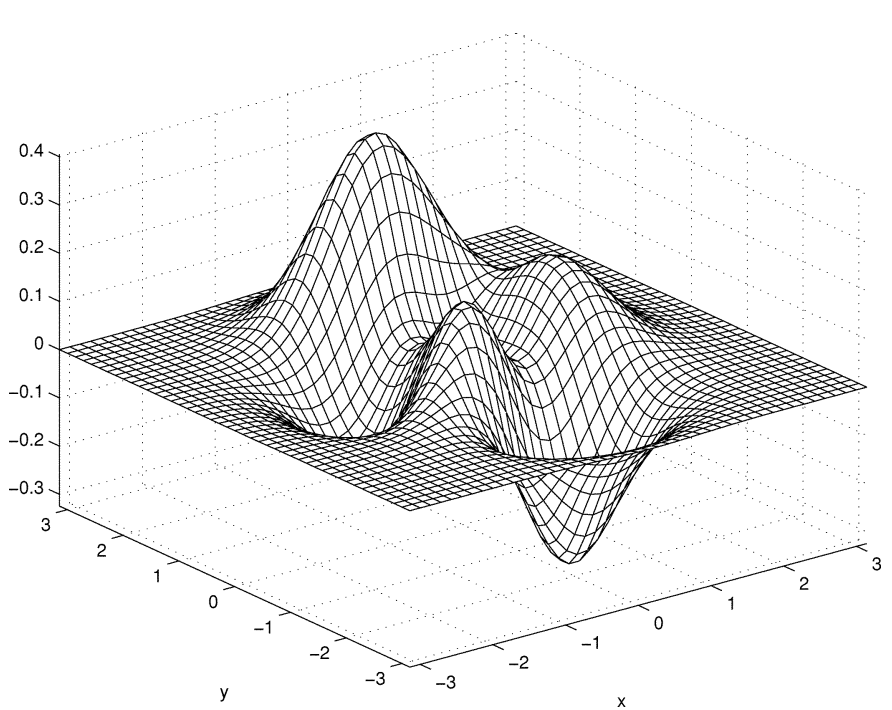

Fig. 5. Scatterer defined by (31) and considered in [6]. Following that reference, this figure displays the graph of $q_{1}(x, y)=-m(x, y)$.

For all nonsmooth scatterers (such as those of Sections IV-C, IV-E) it is necessary to use a Fourier smoothing $m^{F}$ for $m$. We found the value $F=100$ to be adequate in all cases considered here: computation with $F=200$ and much more resolved discretizations (in $\theta$ ) yielded identical results as long as the number of modes $M$ was kept constant. Interestingly, higher values of $F$ degrade the quality of the results, unless finer angular discretizations are used. That is, if an approximation $m^{F}$ is used which is sharper (and, therefore, "less smooth") than it needs to be for a given accuracy in the approximate solution, then, the increased nonsmoothness needs to be resolved by means of a
TABLE XI

ERRORS AND COMPUTING TIMES FOR THE CONFIGURATION OF FIG. 5

$$
\kappa R=\pi
$$

\begin{tabular}{c|c|c|c|c}
\hline$M$ & $N_{r}$ & Restart & Time (sec) & Error (Far Field) \\
\hline 6 & 11 & 3 & 0.09 & $1.2 \cdot 10^{(-4)}$ \\
\hline 8 & 21 & 4 & 0.23 & $4.4 \cdot 10^{(-6)}$ \\
\hline 9 & 41 & 6 & 0.49 & $2.6 \cdot 10^{(-7)}$ \\
\hline 10 & 81 & 7 & 1.05 & $1.6 \cdot 10^{(-8)}$ \\
\hline 12 & 161 & 8 & 2.63 & $9.4 \cdot 10^{(-10)}$ \\
\hline 13 & 321 & 10 & 6.55 & $6.0 \cdot 10^{(-11)}$ \\
\hline 13 & 641 & 10 & 14.24 & $3.7 \cdot 10^{(-12)}$ \\
\hline
\end{tabular}

$$
\kappa R=5 \pi
$$

\begin{tabular}{c|c|c|c|c}
\hline$M$ & $N_{r}$ & Restart & Time (sec) & Error (Far Field) \\
\hline 13 & 11 & 4 & 0.20 & $1.1 \cdot 10^{(-1)}$ \\
\hline 19 & 21 & 7 & 0.57 & $1.7 \cdot 10^{(-3)}$ \\
\hline 21 & 41 & 9 & 1.32 & $1.1 \cdot 10^{(-4)}$ \\
\hline 24 & 81 & 10 & 2.99 & $6.7 \cdot 10^{(-6)}$ \\
\hline 25 & 161 & 13 & 6.96 & $4.2 \cdot 10^{(-7)}$ \\
\hline 26 & 321 & 16 & 18.19 & $2.6 \cdot 10^{(-8)}$ \\
\hline 29 & 641 & 18 & 48.18 & $6.9 \cdot 10^{(-10)}$ \\
\hline
\end{tabular}

$$
\kappa R=9 \pi
$$

\begin{tabular}{c|c|c|c|c}
\hline$M$ & $N_{r}$ & Restart & Time (sec) & Error (Far Field) \\
\hline 26 & 21 & 5 & 0.75 & $1.2 \cdot 10^{(-1)}$ \\
\hline 32 & 41 & 9 & 2.16 & $1.8 \cdot 10^{(-3)}$ \\
\hline 35 & 81 & 12 & 5.05 & $1.1 \cdot 10^{(-4)}$ \\
\hline 38 & 161 & 15 & 13.88 & $6.7 \cdot 10^{(-6)}$ \\
\hline 39 & 321 & 17 & 32.23 & $4.1 \cdot 10^{(-7)}$ \\
\hline 41 & 641 & 20 & 88.91 & $1.1 \cdot 10^{(-8)}$ \\
\hline
\end{tabular}

finer grid in order to avoid introduction of increased integration errors.

The second example tests the performance of our method in a case in which the scatterer contains corner-type singularities (see Fig. 4). The accuracy in these results is comparable to that of the example of the previous section, see Table X. We see that the presence of corners does not affect the performance of our algorithm. 

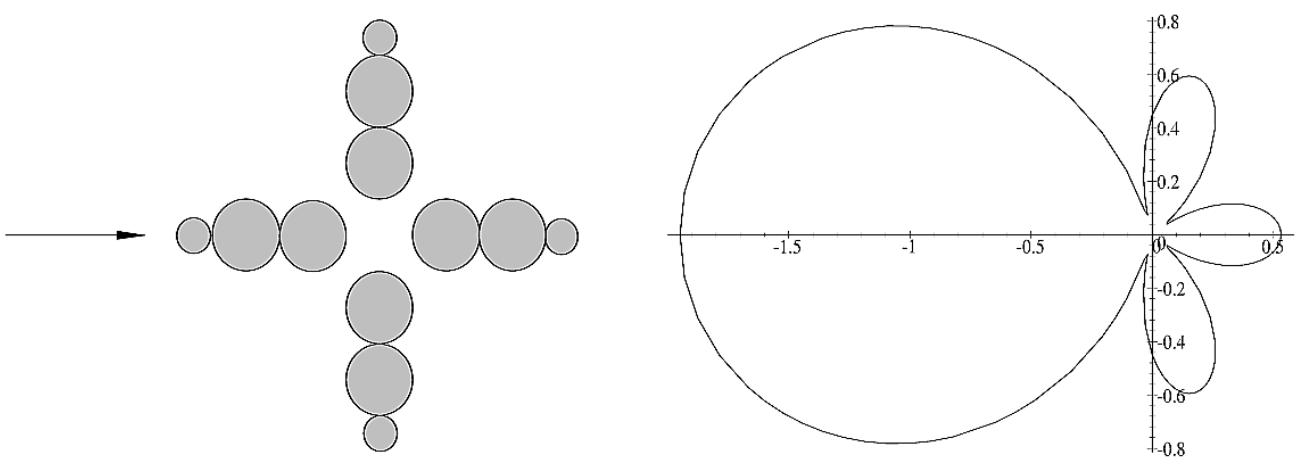

Fig. 6. Left: A scattering configuration containing geometric singularities. Right: Associated far-field pattern; $\kappa R=3$.

TABLE XII

ERRoRs AND TIMINGS FOR THE CONFIGURATION OF FIG. $6: N_{p}=3$ AND $\kappa R=3$, SHOWING A LiNEAR DEPENDENCE OF INTEGRATION TIMES AND OVERHEAD ON $M \cdot N_{r}$

\begin{tabular}{c|c|c|c|c|c|c|c|c}
\hline$n^{2}$ & $M$ & $N_{r}$ & \# Iter & OH(s) & NO(ms) & Tot. Time(s) & NT(ms) & Error (Far Field) \\
\hline 2 & 10 & 23 & 2 & 0.593 & 2.5 & 1.9 & 72 & $1 \cdot 10^{(-3)}$ \\
\hline 2 & 20 & 31 & 2 & 1.273 & 2.0 & 5.0 & 75 & $1 \cdot 10^{(-4)}$ \\
\hline 2 & 30 & 43 & 3 & 2.332 & 1.8 & 12.8 & 67 & $3 \cdot 10^{(-5)}$ \\
\hline 72 & 10 & 19 & 2 & 0.611 & 3.2 & 8.7 & 52 & $3 \cdot 10^{(-1)}$ \\
\hline 72 & 20 & 33 & 3 & 1.666 & 2.5 & 46.9 & 57 & $7 \cdot 10^{(-2)}$ \\
\hline 72 & 30 & 63 & 6 & 4.110 & 2.2 & 268.5 & 57 & $7 \cdot 10^{(-3)}$ \\
\hline
\end{tabular}

\section{An Electrically Large Scatterer}

In a recent study [6] on solution of acoustic inverse problems, configurations were considered for the scatterer defined by

$$
\begin{aligned}
& m(x, y)=-0.15(1-x)^{2} e^{-\left(x^{2}+(y+1)^{2}\right)} \\
& \quad+0.5\left(\frac{x}{5}-x^{3}-y^{5}\right) e^{-\left(x^{2}+y^{2}\right)}+\frac{1}{60} e^{-\left((x+1)^{2}+y^{2}\right)}
\end{aligned}
$$

within the circle of radius $R=\pi$ (see Fig. 5) and with values of $\kappa R$ from $\pi$ to $9 \pi$. We show below a convergence study of our method applied to this problem for $\kappa R=\pi, 5 \pi$ and $9 \pi$; note the quartic convergence on the radial mesh-size which is due to our choice $N_{p}=3$ for the interpolation order (cf. Section IV-A).

Reference [6] does not provide timings for the direct problem solutions; however, the solution of the inverse problem, which includes direct solutions with an accuracy of $10^{(-3)}$ for nine frequencies, is completed in calculations of the order of $120 \mathrm{~s}$ on a Cray C-90 computer. Table XI, on the other hand, shows computations within the same accuracy in computing times of the order of at most $2 \mathrm{~s}$ in a 400-MHz PC.

\section{E. A Scatterer Containing Complex Geometric Singularities}

Finally, we consider the scattering configuration depicted in Fig. 6 formed by twelve touching circles of refractive index $n$, eight of radius 0.5 and four of radius 0.25 , in such a way that the smallest circle containing the scatterer has radius $R=3$. The cusps in this geometry are well known to present severe difficulties to other algorithms. For such a discontinuous refractive index it is necessary to use Fourier smoothing; the value $F=100$ was used here. (Thorough numerical tests, including comparison with the exact solution mentioned later, as well as convergence tests, have shown that the values of the smoothing parameter $F$ we used give converged solutions within the error bars quoted.) Table XII shows convergence studies for $n^{2}=2$ and $n^{2}=72$. Errors in the table (maximum absolute values in the far field) were obtained by comparison with a much more refined discretization: $F=200, M=100$ and $N_{r}=181$ in the case $n^{2}=2$, and $F=200, M=40$, and $N_{r}=301$ for $n^{2}=72$. Near-field errors are generally larger by a factor of 10. As claimed, our algorithm resolves the $n^{2}=2$ configuration with an error of order $10^{(-5)}$ in a 13-s run. The restart parameter required by GMRES was taken to equal 4 in the case $n^{2}=2$ and to equal 40 for $n^{2}=72$. OH denotes the overhead, and NO and NT denote the normalized quantities $\mathrm{NO}=10^{3} \mathrm{OH} /(M$. $\left.N_{r}\right) \mathrm{NT}=10^{3}($ Time $-\mathrm{OH}) /\left(\right.$ Restart $\cdot$ Iter $\left.\cdot M \cdot N_{r}\right)$. In order to insure that these tests give an accurate measure of the error we applied the same procedure to the off-center circle of nonsmooth for which an analytical solution is known. For $n^{2}=2$ using $M=10,20$ and 40 our solver yields errors of $2 \cdot 10^{(-4)}$, $3 \cdot 10^{(-5)}$ and $4 \cdot 10^{(-6)}$, respectively. For $n^{2}=72$ using $M=10$, 20 and 40 modes we obtained errors of $3 \cdot 10^{((0)}, 2 \cdot 10^{(-2)}$ and $2 \cdot 10^{(-3)}$, respectively, in good agreement with the orders of the errors shown in Table XII.

\section{CONCLUSION}

In this paper we have introduced a new high-order integral algorithm for the solution of scattering problems by heterogeneous bodies. As demonstrated in a number of examples, our algorithm runs in $\mathcal{O}(N \log (N))$ operations where $N$ is the number of discretization points, and it produces Far Fields with an error of order $\mathcal{O}\left(h^{3}\right)$ for discontinuous refractive indexes - and much higher convergence rates for smooth refractive indexes. The fast/high-order character of this method allowed us to produce solutions with several digits of accuracy in small computing times, even for large scatterers containing geometric singularities such as corners, edges, and cusps. 

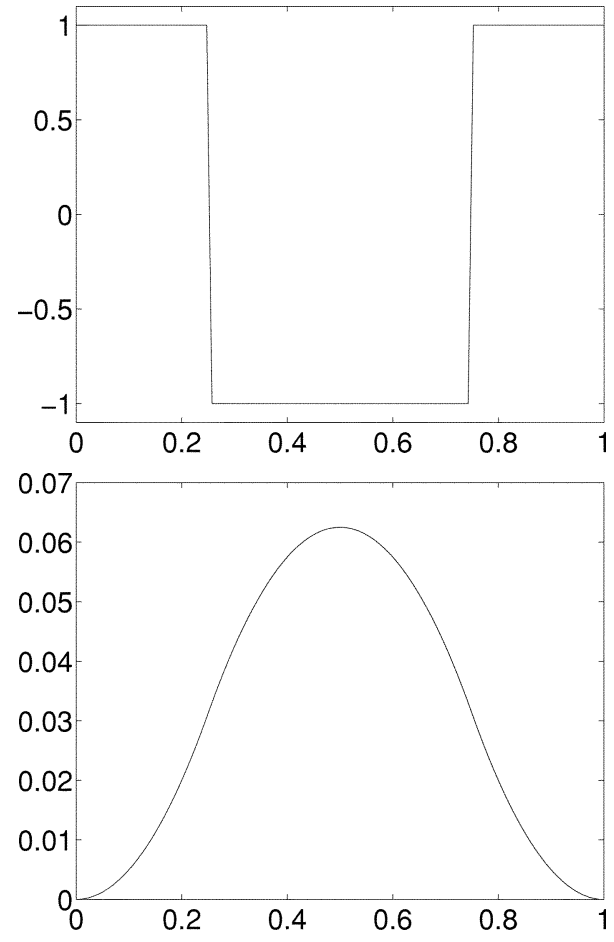

Fig. 7. Functions $f$ and $g$.

\section{APPENDIX \\ ERROR CANCELLATION}

The concept of error cancellation that lies at the basis of the algorithm introduced in this paper may be readily illustrated by means of an elementary numerical example: use of Fourier expansions to produce high-order numerical evaluations of an integral of the form $\int_{0}^{1} f(\theta) g(\theta) d \theta$, where: i) $f$ is a discontinuous periodic function, and where: ii) $g$ is a function which is continuous together with its derivative, but whose second derivative is discontinuous. For this example, we use the functions

$$
f(\theta)=\left\{\begin{array}{cl}
1, & 0 \leq \theta<\frac{1}{4} \\
-1, & \frac{1}{4} \leq \theta<\frac{3}{4} \\
1, & \frac{3}{4} \leq \theta \leq 1
\end{array}\right.
$$

and

$$
g(\theta)=\left\{\begin{array}{cl}
\frac{\theta^{2}}{2}, & 0 \leq \theta<\frac{1}{4} \\
-\frac{\theta^{2}}{2}+\frac{\theta}{2}-0.0625, & \frac{1}{4} \leq \theta<\frac{3}{4} \\
\frac{(\theta-1)^{2}}{2}, & \frac{3}{4} \leq \theta \leq 1
\end{array}\right.
$$

see Fig. 7; we have $\int_{0}^{1} f(\theta) g(\theta) d \theta=-1 / 48$. The discontinuities of $f$ and the degree of smoothness of $g$ correspond, respectively, to those of the refractive index $n$ and the scattered field $u$ for a discontinuous scatterer; in our method, products $u \cdot n$ are integrated over a period of the angular variable for each fixed radius. In analogy with our method, here we proceed to evaluate the integral of $f g$ by replacing $f$ and $g$ by their respective Fourier series truncated to order $F$-including modes between $-F$ and $F$ only - , evaluating their pointwise product, and integrating the result by means of the trapezoidal rule-with $N_{\theta}$ points and mesh-size $h=1 /\left(N_{\theta}-1\right)$. [We emphasize here that the Fourier coefficients of the discontinuous function function $f$ should be accurate, and could not, therefore, be produced by a
TABLE XIII

CONVERGENCE TEST FOR THE EVAlUATION OF $\int_{0}^{1} f(\theta) g(\theta) d \theta$ AS A FunCTION THE NUMBER OF MODES $N$ USED FOR $f$ AND $g$

\begin{tabular}{c|c|c|c}
\hline$F$ & $N_{\theta}$ & Absolute Error & Ratio \\
\hline 2 & 4 & $3.0 \cdot 10^{(-4)}$ & \\
\hline 4 & 8 & $4.8 \cdot 10^{(-5)}$ & 6.3 \\
\hline 8 & 16 & $6.5 \cdot 10^{(-6)}$ & 7.4 \\
\hline 16 & 32 & $8.3 \cdot 10^{(-7)}$ & 7.8 \\
\hline 32 & 64 & $1.0 \cdot 10^{(-7)}$ & 8.3 \\
\hline
\end{tabular}

simple integration rule. Fortunately, it is not hard to produce accurate Fourier coefficients for $f$, as it is generally the case for the angular Fourier coefficients of a given distribution $n$ of refractive indexes: either closed expressions or simple 1-D high-order integration rules "by-pieces" can be used.] The accuracies resulting from these operations are displayed in Table XIII. We see that the error in the approximate integral is of the order $h^{3}$ (since the "Ratio" of the absolute error for $N_{\theta}=2^{n-1}$ to that resulting for $N_{\theta}=2^{n}$ is approximately equal to 8 ) in spite of both, the Gibbs phenomenon and the low order convergence of the series for the discontinuous function $f$. This cancellation of errors can be explained through consideration of the error arising in the zero-th order coefficient of the function $f g$ as a result of the truncations used, see [4]. Naturally, even higher order convergence results for smoother functions $f$ and $g$ (or $n$ and $u$ ). We point out, however, that, even in the most singular case considered here, it suffices to use 64 points to produce results with an accuracy better than full single precision. Tight error estimates for the numerical method presented in this paper are given in [4].

\section{ACKNOWLEDGMENT}

The authors wish to thank E. M. Hyde for providing the numerical implementation which was used to produce the tables concerning angular integration.

\section{REFERENCES}

[1] J. J. Bowman, T. B. A. Senior, and P. L. E. Uslenghi, Electromagnetic and Acoustic Scattering by Simple Shapes. Bristol, PA: Hemisphere, 1987.

[2] N. N. Bojarski, "The k-space formulation of the scattering problem in the time domain," J. Opt. Soc. Amer, vol. 72, pp. 570-584, 1982.

[3] A. Brandt and A. A. Lubrecht, "Multilevel matrix multiplication and fast solution of integral equations," J. Comp. Phys., vol. 90, pp. 348-370, 1990.

[4] O. P. Bruno and E. M. Hyde, "High-order Fourier approximation in scattering by two-dimensional, inhomogeneous media," Siam J. Numer. Anal., to be published.

[5] P. Bruno, E. M. Hyde, and F. Reitich, "An accelerated solver for penetrable obstacle scattering with tunable order of convergence," SIAM J. Sci. Computing, submitted for publication.

[6] Y. Chen, "Inverse scattering via Heisenberg's uncertainty principle," Inverse Problems 13, pp. 253-282, 1997.

[7] D. Colton and R. Kress, Inverse Acoustic and Electromagnetic Scattering Theory. New York: Springer-Verlag, 1992.

[8] A. Greenbaum, L. Greengard, and G. McFadden, "Laplace equation and the Dirichlet-Neumann map in multiply connected domains," J. Comp. Phys., vol. 105, pp. 267-278, 1993.

[9] L. Greengard and V. Rokhlin, "A fast algorithm for particle simulations," J. Comp. Phys., vol. 73, pp. 325-348, 1987.

[10] L. Greengard and J. Lee, "A direct adaptive Poisson solver of arbitrary order accuracy," J. Comp. Phys., vol. 125, pp. 415-424, 1996.

[11] E. Isaacson and H. B. Keller, Analysis of Numerical Methods. New York: Wiley, 1966.

[12] H. C. Van De Hulst, Light Scattering by Small Particles. New York: Wiley, 1957. 
[13] A. Kirsch and P. Monk, "An analysis of the coupling of finite-element and Nyström methods in acoustic scattering," IMA J. Numer. Anal., vol. 14, pp. 523-544, 1994.

[14] E. Martensen, "Über eine methode zum räumlichen Neumannschen problem mit einer anwendung für torusartige berandungen," Acta Math., vol. 109, pp. 75-135, 1963.

[15] W. H. Press, S. A. Teukolsky, W. T. Vetterling, and B. P. Flannery, $N u$ merical Recipes. Cambridge, U.K.: Cambridge Univ. Press, 1992.

[16] V. Rokhlin, "Rapid solution of integral equations of classical potential theory," J. Comp. Phys., vol. 60, pp. 187-207, 1985.

[17] _ - "Rapid solution of integral equations of scattering theory in two dimensions," J. Comp. Phys., vol. 86, pp. 414-439, 1990.

[18] P. Zwamborn and P. Van den Berg, "Three dimensional weak form of the conjugate gradient FFT method for solving scattering problems," IEEE Trans. Microwave Theory Tech., vol. 40, no. 9, pp. 1757-1766, 1992.

[19] O. P. Bruno and A. Sei, "A fast high-order solver for em scattering from complex penetrable bodies: TE case," IEEE Trans. Antennas Propagat., vol. 48, pp. 1862-1863, Dec 2000.

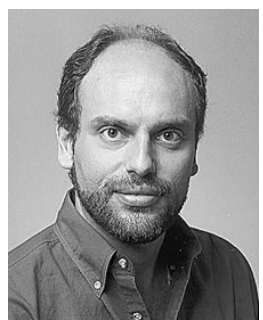

Oscar P. Bruno received the Ph.D. degree from the Courant Institute of Mathematical Sciences, New York University, in 1989.

Following graduation, he held a position for two years as Visiting Assistant Professor with the University of Minnesota, and then joined the faculty of the Georgia Institute of Technology (Georgia Tech), where he held positions as Assistant Professor and Associate Professor. After a four-year period with Georgia Tech, he joined the faculty of the California Institute of Technology (Caltech), Pasadena, where he is now Professor in the Department of Applied Mathematics, having served as Executive Officer during 1998-2000. His research interests are in the areas of optics, elasticity and electromagnetism, remote sensing, radar, overall electromagnetic and elastic behavior of materials (solid, fluids, composites materials, multiple-scale geometries), and solid-to-solid phase transitions.

Dr. Bruno graduated with a Friedrichs Award for an outstanding dissertation in mathematics from the Courant Institute. He is the recipient of a Young Investigator Award from the National Science Foundation and a Sloan Foundation Fellowship.

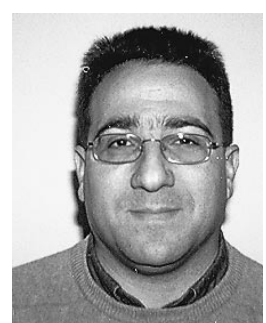

Alain Sei was born in 1965. He received the B.S. and M.S. degrees in mathematics in 1986 and 1988, respectively, from the University of Pau, Pau, France. He received the Ph.D. degree in applied mathematics from the University of Paris Dauphine, Paris, France, in 1991.

From 1986 to 1991, he was a Researcher with the French Institute of Petroleum (IFP). From 1993 to 1995, he was a Research Scientist with Rice University, Houston, TX. In 1995, he took a Postdoctoral Fellowship in applied mathematics at the California Institute of Technology (Caltech), Pasadena, and continued his research until joining TRW (currently Northrop Grumman Space Technology) in 1998 as a Senior Engineer. His areas of interest include mathematical modeling and numerical techniques for direct and inverse wave propagation problems. 